\title{
Aplicación Del Análisis Envolvente De Datos En Las Universidades Autónomas De Bolivia. Caso Carreras Ciencias Empresariales
}

\author{
Mgs. Wendy Roxana Jordán Minaya \\ Universidad Tecnica De Oruro - Bolivia \\ PhD. Gabith Miriam Quispe Fernández \\ Universidad Nacional De Chimborazo- Ecuador
}

Doi: 10.19044/esj.2017.v13n28p155 URL:http://dx.doi.org/10.19044/esj.2017.v13n28p155

\begin{abstract}
The technical efficiency is a tool that allows to know the situation in which an institution is located. In this context, the objective of the research is to determine the technical efficiency indices of the years 2014 and 2015 in order to carry out a comparative analysis and identify their causes in the careers related to the Economic and Business Sciences of the State Autonomous Public Universities Plurinacional de Bolivia through the application of Data Envelopment Analysis (DEA) as a tool for analysis of technical efficiency. The research constitutes the first work of analysis of university efficiency. 69 studies belonging to 11 Universities of the Bolivian University System are considered as study population. The results show that there are careers with high indices of technical efficiency; on average, there is an increase in technical efficiency between 2014 and 2015; the number of students, graduates and teachers are what determine the rates of technical efficiency in careers.
\end{abstract}

Keywords: Efficiency, University, Growing Yields, Variable Yields, Education

\section{Resumen}

La eficiencia técnica es una herramienta que permite conocer la situación en la cual se encuentra una institución. En ese contexto, la investigación tiene el objetivo de determinar los índices de eficiencia técnica de los años 2014 y 2015 a fin de realizar un análisis comparativo e identificar sus causas en las Carreras relacionadas con las Ciencias Económicas y Empresariales de las Universidades Públicas Autónomas del Estado Plurinacional de Bolivia a través de la aplicación del Análisis Envolvente de Datos (DEA) como una herramienta de análisis de la 
eficiencia técnica. La investigación se constituye en el primer trabajo de análisis de eficiencia universitaria. Se considera como población de estudio 69 carreras pertenecientes en 11 Universidades del Sistema de Universidades Bolivianas. Los resultados muestran que existen carreras con altos índices de eficiencia técnica; en promedio existe un incremento de la efíciencia técnica entre el año 2014 y 2015; el número de alumnos, titulados y docentes son los que determinan los índices altos de eficiencia técnica en las carreras.

Palabras-claves: Eficiencia, Universidad, Rendimientos crecientes, Rendimientos variables, Educación

\section{Introducción}

Abordar el estudio de las universidades significa identificar cual es el reto a la cual se enfrenta, Jimenes (2006, pág. 191) menciona que "uno de los más grandes retos que hoy enfrentan las universidades es encontrar las formas y los mecanismos para adaptar sus funciones a los nuevos modos de producción y difusión del conocimiento". Asi, si se considera que la educación es uno de los factores de desarrollo de un país, como lo ratifica Neira (2010, pág. 1) cuando menciona que "educación de la población es un factor condicionante del desarrollo económico" y es un "factor clave para aumentar la competitividad internacional de su estructura productiva y acceder a un nivel de alta expansión económica de largo plazo"(Moreno-Brid \& Ruiz-Nápoles, 2010, pág. 172-176). Lo que significa que las universidades públicas se convierten en "las instituciones en las que se forman las personas y se realiza la mayor parte de la investigación y el desarrollo científico y tecnológico de cada país" (Moreno-Brid, 2009, pág. 12) convirtiendose "la educación una estrategia viable para asegurar un desarrollo sostenible ya que genera capital humano y social, y en consecuencia desarrollo humano, lo cual mejora la productividad, la convivencia social, avances en la investigación científica y tecnológica, entre otros recursos" (García \& Talancón, 2008, pág. 7). Entonces, las tendencias de la universidad es responder a la expanción, diversificación, restricciones financieras, calidad academica y administrativa y a la internacionalización.

En ese contexto en el Estado Plurinacional de Bolivia (EPB) la educación superior en las Universidades publica viene regulada su funcionamiento por normas, como: 1) la Constitución Política del Estado Plurinacional de Bolivia promulgado el 09 de febrero del 2009; 2) El Estatuto Orgánico de la Universidad Boliviana (CEUB C. E., Estatuto Orgánico de la Universidad Boliviana, 2013) Aprobado por Resolución $\mathrm{N}^{\circ}$ 026/2013 del XII Congreso Nacional de Universidades; 3) se reglamenta el Sistema Nacional de Planificación del Sistema de la Universidad Boliviana; 
aprobado en el XII Congreso Nacional de Universidades por Resolución Nro. 029/2014; y 4) el Plan Nacional de Desarrollo Universitario (PNDU) 2014 - 2018 aprobado en la II Conferencia Nacional Ordinaria de Universidades, según Resolución $\mathrm{N}^{\circ}$ 03/2014, en cambio las universidades privadas bolivianas son reguladas por el Reglamento General de Universidades Privadas (RGUP), aprobado por Decreto Supremo $\mathrm{N}^{\circ} 1433$ del 2012, y como órgano ejecutor por el Ministerio de Educación, ya que la apertura de programas académicos en las universidades privadas y su desarrollo institucional es regulado por el Ministerio de Educación (Ministerio de Educación, 2012, pág. 2). El financiamiento económico y su administración para el funcionamiento de las Universidades estatales bolivianas, se encuentran reguladas bajo la Ley $\mathrm{N}^{\circ} 1178$ de Administración y Control Gubernamental.

Uno de los aspectos más importantes de la educación superior es lograr una educación de calidad, en el caso de las Universidades Públicas Bolivianas la calidad universitaria es un objetivo fundamental para lograr el desarrollo, como se menciona en el Estatuto Orgánico de la Universidad Boliviana y que responde al Plan Nacional de Desarrollo Estratégico (Plan Nacional de Desarrollo Universitario 2014 - 2018, pág. 74). En este contexto, a pesar de que el "concepto de calidad en la educación superior no existe como tal, sino como un término de referencia de carácter comparativo en el cual algo puede ser mejor o peor que otro, dentro de un conjunto de elementos homologables, o en comparación con cierto patrón de referencia real o utópico- previamente determinado" (González y Ayarza, 1990), la calidad universitaria está "ligada a la pertinencia social, así como a la preparación y compromiso de los profesores e investigadores" (Miranda, 2001, pág. 13), además "es una meta que se alcanza mediante un proceso en el que participan activamente los docentes y estudiantes... este proceso implica también el máximo nivel de competencia académica en todas las disciplinas que se estudien" (Miranda, 2001, pág. 16). Por tanto, "sólo se puede establecer que una institución es mejor que otra cuando son homólogas en sus fines, concordantes en su misión y se encuentran en un contexto similar (González ,2008, págs. 252 - 253) y que la " coherencia entre input y procesos con los productos, define la calidad de la educación universitaria como eficiencia" (Orden Hoz, Asensio Muñoz, Biencinto López, \& González, 2007, pág. 8), lo que significa que la calidad implica eficiencia y eficacia educativa, por tanto es de importancia la medición de la eficiencia universitaria.

Los distintos estudios realizados de la medición de la efíciencia educativa universitaria a traves del DEA estan relaionados con aquellos aplicados a: 1) Universidades Estatales a través de Larrán y García (2015); Escorcia, Visbal \& Agudelo (2014); Larrán y García (2015); Amariles y 
Soto-Mejía (2015); Cáceres, Kristjanpoller \& Tabilo (2013); Ramírez \& Alfaro (2013); Coria (2011); Vásquez (2011); García \& Larrán (2008); 2) Instituciones Educativas realizado por Escorcia, Visbal y Agudelo (2014); estudios de eficiencia en departamentos Martín (2008) y 3) Profesores (Visbal, Mendoza \& García, 2015) estos con distintos objetivos, como se puede obsevar en el Cuadro 1.

Cuadro 1. Estudios realizados sobre la eficiencia educativa en países de Europa, Australia y América Latina

\begin{tabular}{|c|c|c|c|c|}
\hline AUTOR & ESTUDIO & OBJETIVO & $\begin{array}{l}\text { APLICADO } \\
\text { EN: }\end{array}$ & $\begin{array}{l}\text { CLASIF } \\
\text { ICACIÓ } \\
\quad \mathrm{N} \\
\text { INSTIT } \\
\text { UCION } \\
\quad \text { AL } \\
\end{array}$ \\
\hline $\begin{array}{c}\text { Jorge Manuel } \\
\text { Larrán y Ángel } \\
\text { García Correas } \\
\text { (2015) }\end{array}$ & $\begin{array}{c}\text { ¿Influyen los modelos de } \\
\text { financiación autonómicos en } \\
\text { la eficiencia de las } \\
\text { Universidades Públicas } \\
\text { españolas? }\end{array}$ & $\begin{array}{c}\text { Estudiar la eficiencia de las } \\
\text { Universidades Públicas } \\
\text { españolas bajo las dimensiones } \\
\text { separadas de docencia, } \\
\text { investigación y transferencia } \\
\text { de conocimientos } \\
\end{array}$ & $\begin{array}{l}47 \\
\text { Universidades } \\
\text { Públicas } \\
\text { Presenciales } \\
\text { españolas }\end{array}$ & Pública \\
\hline $\begin{array}{c}\text { Abbott M. y } \\
\text { Doucouliagos C. } \\
\text { (2003) }\end{array}$ & $\begin{array}{c}\text { La Eficiencia de las } \\
\text { Universidades de Australia: } \\
\text { Análisis Envolvente de } \\
\text { Datos }\end{array}$ & $\begin{array}{l}\text { Evaluar la eficiencia de las } \\
\text { Universidades de Australia }\end{array}$ & $\begin{array}{l}\text { Universidades } \\
\text { de Australia }\end{array}$ & Pública \\
\hline $\begin{array}{l}\text { Rolando Escorcia } \\
\text { Caballero, } \\
\text { Delimirio Visbal } \\
\text { Cadavid y José } \\
\text { María Agudelo } \\
\text { Toloza (2014) }\end{array}$ & $\begin{array}{c}\text { Eficiencia en las } \\
\text { Instituciones educativas } \\
\text { públicas de la ciudad de } \\
\text { Santa Marta (Colombia) } \\
\text { mediante "Análisis } \\
\text { Envolvente de Datos" }\end{array}$ & $\begin{array}{l}\text { Determinar los índices de la } \\
\text { eficiencia técnica ET; } \\
\text { puramente técnica ETP y de } \\
\text { escala (EE) de las Unidades } \\
\text { Educativas Distritales (UED) } \\
\text { de Santa Marta a partir de la } \\
\text { técnica análisis envolvente de } \\
\text { datos y establecer protocolos } \\
\text { de mejora para las instituciones } \\
\text { que resultaran ineficientes }\end{array}$ & $\begin{array}{l}44 \text { Instituciones } \\
\text { Educativas de } \\
\text { Santa Marta } \\
\text { (Colombia) }\end{array}$ & Pública \\
\hline $\begin{array}{l}\text { Delimiro Visbal } \\
\text { Cadavid, Adel } \\
\text { Mendoza } \\
\text { Mendoza y Karen } \\
\text { Corredor Carrasal } \\
\text { (2015) }\end{array}$ & $\begin{array}{l}\text { Evaluación del desempeño } \\
\text { docente mediante un análisis } \\
\text { envolvente de datos un } \\
\text { estudio de caso. }\end{array}$ & $\begin{array}{l}\text { Evaluar el desempeño de los } \\
\text { docentes universitarios (en } \\
\text { cada uno de los cursos }\end{array}$ & $\begin{array}{l}\text { Profesores y sus } \\
\text { asignaturas (405 } \\
\text { Unidades } \\
\text { profesor/asignat } \\
\text { ura) }\end{array}$ & Pública \\
\hline $\begin{array}{l}\text { Juan M. Amariles } \\
\text { y José A. Soto } \\
\text { Mejía (2015) }\end{array}$ & $\begin{array}{l}\text { Análisis de Sensibilidad de } \\
\text { los resultados del modelo de } \\
\text { gestión SUE (Sistema } \\
\text { Universitario Estatal) basado } \\
\text { en el análisis envolvente de } \\
\text { datos. }\end{array}$ & $\begin{array}{c}\text { Propuesta de un modelo } \\
\text { alternativo basado en el } \\
\text { Análisis Envolvente de Datos, } \\
\text { que permite calcular el } \\
\text { desempeño de las } \\
\text { Universidades del SUE }\end{array}$ & $\begin{array}{l}32 \\
\text { Universidades } \\
\text { Estatales del } \\
(2012-2014)\end{array}$ & Pública \\
\hline
\end{tabular}




\begin{tabular}{|c|c|c|c|c|}
\hline $\begin{array}{l}\text { Hernán Cáceres } \\
\text { V.; Werner } \\
\text { Kristjanpoller R. y } \\
\text { Jorge Tabilo A. } \\
\text { (2014) }\end{array}$ & $\begin{array}{l}\text { Análisis de la Eficiencia } \\
\text { Técnica y su relación con los } \\
\text { resultados de la evaluación } \\
\text { de desempeño en } \\
\text { Universidad Chilena. }\end{array}$ & $\begin{array}{l}\text { Medir la eficiencia técnica de } \\
\text { las Universidades Académicas } \\
\text { de la Universidad Chilena } \\
\text { utilizando el método del DEA }\end{array}$ & $\begin{array}{c}15 \\
\text { Universidades } \\
\text { Chilenas }\end{array}$ & Pública \\
\hline $\begin{array}{l}\text { Raquel Martín } \\
\text { (2008) }\end{array}$ & $\begin{array}{c}\text { La Medición de la Eficiencia } \\
\text { Universitaria: Una aplicación } \\
\text { del Análisis Envolvente de } \\
\text { Datos }\end{array}$ & $\begin{array}{l}\text { Analizar la Eficiencia Técnica } \\
\text { en los departamentos de la } \\
\text { Universidad de La Laguna }\end{array}$ & $\begin{array}{l}62 \text { Carreras de } \\
\text { la Universidad } \\
\text { de La Laguna }\end{array}$ & Pública \\
\hline $\begin{array}{l}\text { Patricio E. } \\
\text { Ramirez y Jorge } \\
\text { L. Alfaro (2013) }\end{array}$ & $\begin{array}{c}\text { Evaluación de la Efíciencia } \\
\text { de las Universidades } \\
\text { pertenecientes al Consejo de } \\
\text { Rectores de las } \\
\text { Universidades Chilenas : } \\
\text { Resultados de un Análisis } \\
\text { Envolvente de Datos }\end{array}$ & $\begin{array}{l}\text { Estimar la eficiencia de las } \\
\text { Universidades pertenecientes } \\
\text { al Consejo de Rectores de las } \\
\text { Universidades Chilenas }\end{array}$ & \begin{tabular}{c|}
25 \\
Universidades \\
pertenecientes al \\
Consejo de \\
Rectores de las \\
Universidades \\
Chilenas
\end{tabular} & Pública \\
\hline $\begin{array}{l}\text { María Marta } \\
\text { Coria (2011) }\end{array}$ & $\begin{array}{l}\text { Eficiencia Técnica de las } \\
\text { Universidades Argentinas de } \\
\text { gestión estatal }\end{array}$ & $\begin{array}{c}\text { Medir la eficiencia técnica de } \\
\text { las Universidades Argentinas } \\
\text { de gestión estatal }\end{array}$ & $\begin{array}{l}\text { 38 } \\
\text { Universidades } \\
\text { Argentinas de } \\
\text { gestión estatal }\end{array}$ & Pública \\
\hline $\begin{array}{c}\text { Angélica María } \\
\text { Vásquez Rojas; } \\
\text { Directora Dra. } \\
\text { Carmen Pérez } \\
\text { Esparella (2011) }\end{array}$ & $\begin{array}{l}\text { Eficiencia Técnica y cambio } \\
\text { de productividad en la } \\
\text { Educación Superior Pública. } \\
\text { Un estudio aplicado al Caso } \\
\text { Español }(2000-2009)\end{array}$ & $\begin{array}{l}\text { Evaluar la eficiencia } \\
\text { productiva de las universidades } \\
\text { se enfrenta a ciertas } \\
\text { adversidades muchas de las } \\
\text { cuales comúnmente están } \\
\text { presentes en la producción de } \\
\text { servicios; así como otras muy } \\
\text { concretas del ámbito } \\
\text { educativo. }\end{array}$ & $\begin{array}{c}47 \\
\text { Universidades } \\
\text { Públicas } \\
\text { Presenciales } \\
\text { españolas }\end{array}$ & Pública \\
\hline $\begin{array}{l}\text { Angel García } \\
\text { Correas y Manuel } \\
\text { Larrán Jorge } \\
(2008)\end{array}$ & $\begin{array}{l}\text { Financiación y Eficiencia de } \\
\text { las Universidades Públicas } \\
\text { Españolas: Un Estudio } \\
\text { Empírico }\end{array}$ & $\begin{array}{l}\text { Estudiar la eficiencia de las } \\
\text { universidades públicas } \\
\text { españolas bajo las dimensiones } \\
\text { separadas de docencia, } \\
\text { investigación y transferencia } \\
\text { del conocimiento; así como } \\
\text { relacionar la misma con los } \\
\text { niveles de financiación que } \\
\text { recibe cada Universidad }\end{array}$ & $\begin{array}{c}48 \\
\text { Universidades } \\
\text { Públicas } \\
\text { Españolas (2006 } \\
\text { - 2007) }\end{array}$ & Pública \\
\hline
\end{tabular}

Fuente: Elaboración propia con base en estudios de Larrán y García, 2015; Escorcia , Visbal y Agudelo, 2014; Abbott y Doucouliagos, 2003; Amariles y Soto, 2015; Cáceres, Kristjanpoller y Tabilo, 2013; Ramírez y Alfaro, 2013; Coria, 2011; Vásquez, 2011; García y Larrán, 2008; Escorcia, Visbal y Agudelo, 2014; Martín, 2008; Visbal, Mendoza y García, 2015. 
Después de revisar los estudios realizados en países de América Latina, Europa y Australia se identifica que existen dos tipos de análisis de eficiencia: 1) el análisis de costo - beneficio y; 2) el análisis de la eficiencia en términos de outputs e inputs. Como resultado de ello uno de los enfoque más utilizados; es el análisis de frontera; que estudia la eficiencia de unidad respecto del mejor comportamiento mostrando por algunas de las unidades de la muestra que formarán parte de la frontera, donde las ineficiencias que se encuentran por debajo de la frontera de producción o por encima de la frontera de coste; este análisis permite considerar varios inputs y outputs. Siendo un paso previo a la determinación de la función de producción. De la misma manera el análisis de frontera adopta dos tipos de análisis, una paramétrica y otra no paramétrica.

El análisis de la frontera paramétrica, se refiere a una forma funcional específica para la función de costo o beneficio de las entidades; en cuanto a la estimación siempre y cuando se conozca a priori la función que relaciona las variables pertinentes. La ventaja de este método, es que está basado en procedimientos estadísticos, que toman en cuenta la presencia de ruido aleatorio, generado por errores de medición. Por consiguiente se presentan tres métodos paramétricos, que son: 1) la Frontera Estocástica (FE) o frontera econométrica, que consiste en la estimación de una frontera del entorno; 2) la Distribution Free Approach (DFA), este método no requiere suponer una distribución de probabilidad específica para la ineficiencia; por lo que se puede encontrar el promedio del término de error para cada entidad a través del tiempo utilizando datos de panel. Para ello, el cálculo muestra la desviación de cada entidad respecto a la frontera, ya que el promedio del ruido aleatorio, se supone, debe ser cero a través del tiempo. Encontrándose de esta manera la ineficiencia sin necesidad de suponer una determinada distribución; y 3) el método de la Frontera Gruesa (FG), que es el asignar a los individuos de la muestra en dos grupos, siendo el primero, el que se consideran más eficientes y el segundo grupo el que se consideran más ineficientes, estimándose posteriormente dos fronteras, una para cada grupo, por último se comparan las diferencias entre ambos.

En cambio el análisis de frontera no paramétrica se basa en un procedimiento básico, fundamentado en técnicas de optimización lineal, que consiste en el cálculo de envoltura convexa alrededor de los puntos que representan a cada firma en el espacio de producción, insumos y costos. Donde la envoltura se asimila a la frontera eficiente. Entre los métodos más conocidos son el Análisis Envolvente de Datos (DEA) y el Free Disposal Hull (FDH). Por las características de la educación y la existencia de un grado de homogenidad de datos entre ellas se utiliza para encontrar los índices de eficiencia el modelo de Análisis Envolvente de Datos (Data Envelopment Analysis- DEA) a fin de encontrar la eficiencia desde la 
perspectiva del modelo outputs rendimientos con escala constante de Charnes, Cooper y Rhoders (CCR) y rendimientos con escala variables de Banker, Charnes y Cooper (BCC).

En el Cuadro 2, se aprecia un detalle de las "variables proxy utilizadas en la mayoría de los trabajos existentes en la literatura..."; que fueron utilizados para medir los outputs, por lo que se dividen, según las principales funciones de la universidad (Vázquez, 2011, p. 102).

Cuadro 2: Variables proxy del output Docentes

\begin{tabular}{|c|c|}
\hline Docencia & Autores \\
\hline $\begin{array}{l}\text { 1. Número de titulos superiores adjudicados para postgrado } \\
\text { (número de titulados) }\end{array}$ & Athanasoupolus y Shale, 1997; Flegg et al. 2004 \\
\hline $\begin{array}{l}\text { 2. Número de diplomados otorgados a nivel de grado y } \\
\text { postgrado (expedidos el último curso) }\end{array}$ & Abbott y Doucouliagos, 2003 \\
\hline 3. Número de alumnos graduados & $\begin{array}{l}\text { Ahn y Seiford, 1993; Athanasoupolus y Shale, 1997; } \\
\text { Gómez Sancho, 2005; Hernangómez et al, 2007; } \\
\text { Agasisti y Johnes, 2009; Agasisti y Pérez Esparrells, } \\
2010 .\end{array}$ \\
\hline 4. Estudiantes de grado matriculados ETC & $\begin{array}{l}\text { Ahn y Seiford, 1993; Coelli, Prasada y Battesse, 1998; } \\
\text { Avkiran, 2001; Abbott y Douconliagos, 2003; Johnes } \\
\text { et al , 2005; Thanasoullis et al. } 2011\end{array}$ \\
\hline $\begin{array}{l}\text { 5. Número de alumnos matriculados en centros propios en } 1^{\circ} \mathrm{y} \\
2^{\circ} \text { ciclo }\end{array}$ & $\begin{array}{l}\text { Ahn, Charnes y Cooper, 1988; Parrellada y Duch, } \\
\text { 2006; Duch, } 2006\end{array}$ \\
\hline 6. Estudiantes de postgrado matriculados ETC & $\begin{array}{l}\text { Avkiran, 2001; Johnes et al, 2005; Thanasoullis et al. } \\
2011\end{array}$ \\
\hline 7. Tasa de retención, progreso o éxito de estudiantes & $\begin{array}{l}\text { Breu y Raab, 1994; Avkiran, 2001, Alfonso y Santos, } \\
\text { 2008; Duch-Brown y Vilalta, 2010 }\end{array}$ \\
\hline 8. Tasa de empleo de graduados a tiempo completo & Avkiran, 2001 \\
\hline 9. Pago de matrícula de extranjeros & Avkiran, 2001 \\
\hline 10. Pago de matrícula de postgrado de no extranjeros & Avkiran, 2001 \\
\hline 11. Número de programas ofertados de grado y postgrado & Abbott y Doucouliagos, 2003 \\
\hline 12. Número de matriculados en doctorado & $\begin{array}{l}\text { Ahn, Charnes y Cooper, } 1988 \text { y 1989; Parrellada y } \\
\text { Duch, 2006; Duch, } 2006\end{array}$ \\
\hline 13. \% de alumnos que terminan respecto a los matriculados & Hernangómez et al, 2007 \\
\hline 14. Porcentaje de dobles titulaciones/número de titulaciones & Hernangómez et al, 2007 \\
\hline $\begin{array}{l}\begin{array}{l}\text { 15. Número de titulaciones de segundo ciclo/número de } \\
\text { titulaciones }\end{array} \\
\end{array}$ & Hernangómez et al, 2007 \\
\hline $\begin{array}{|ccccc|}\begin{array}{c}\text { 16. Número de programas de } \\
\text { departamentos }\end{array} & \text { doctorado/número de } \\
\end{array}$ & Hernangómez et al, 2007 \\
\hline $\begin{array}{l}\text { 17. Número de alumnos extranjeros entrantes/número de } \\
\text { alumnos matriculados }\end{array}$ & Hernangómez et al, 2007 \\
\hline $\begin{array}{l}\text { 18. Número de alumnos Erasmus salientes/número de alumnos } \\
\text { matriculados }\end{array}$ & Hernangómez et al, 2007 \\
\hline $\begin{array}{l}\text { 19. Número de provincias distintas donde hay campus/número } \\
\text { de provincias donde hay campus en España }\end{array}$ & Hernangómez et al, 2007 \\
\hline $\begin{array}{l}\text { 20. Número acumulativo de certificados de doctorado otorgados } \\
\text { en los años 2002-2003 por } 100 \text { profesores en cada DMU } \\
\text { (universidad) }\end{array}$ & Alfonso y Santos, 2008 \\
\hline $\begin{array}{l}\text { 21. } \mathrm{N}^{\circ} \text { de alumnos matriculados en cada universidad } \\
\mathrm{N}^{\circ} \text { de alumnos graduados en cada universidad }\end{array}$ & Larrán y García, 2015 \\
\hline 22. Docentes asignatura de la Facultad de Ingeniería & Visbal, Mendoza y Corredor, 2015 \\
\hline
\end{tabular}




\begin{tabular}{|l|l|}
\hline Docencia & Autores \\
\hline $\begin{array}{l}\text { 23. Cantidad de inscripciones de pregrado, Matrícula de } \\
\text { estudiantes nuevos de pregrado, Ingresos propios, } \\
\text { Publicaciones e Ingresos por matrícula de pregrado }\end{array}$ & Cáceres, Kristjan y Tabilo, 2013 \\
\hline $\begin{array}{l}\text { 24. Porcentaje de Profesores buena valoración encuesta } \\
\mathrm{N}^{\circ} \text { de créditos impartidos por departamento } \\
\mathrm{N}^{\circ} \text { de matriculados en } 1^{\circ} \text { y } 2^{\circ} \text { ciclo }\end{array}$ & Martín, 2008 \\
$\mathrm{N}^{\circ}$ alumnos de $3^{\circ}$ ciclo por departamento & \\
\hline 25. $\mathrm{N}^{\circ}$ de estudiantes matriculados & Ramirez y Alfaro, 2013 \\
\hline $\begin{array}{l}\text { 26. Cargos docentes exclusivos equivalentes } \\
\text { Graduados }\end{array}$ & Coria, 2011 \\
\hline $\begin{array}{l}\text { 25. Número de alumnos graduados en } 1^{\circ} \text { y } 2^{\circ} \text { ciclo } \\
\text { Número de artículos ISI publicados al año por } \\
\text { universidad }\end{array}$ & Vázquez, 2011 \\
\hline $\begin{array}{l}\text { 26. Número de alumnos matriculados en cada } \\
\text { Universidad } \\
\text { Número de alumnos graduados en cada Universidad }\end{array}$ & García y Larrán, 2008 \\
\hline
\end{tabular}

Fuente: Elaboración propia con base en estudios de Larrán y García, 2015; Escorcia , Visbal y Agudelo, 2014; Abbott y Doucouliagos, 2003; Amariles y Soto, 2015; Cáceres, Kristjanpoller y Tabilo, 2013; Ramírez y Alfaro, 2013; Coria, 2011; Vásquez, 2011; García y Larrán, 2008; Escorcia, Visbal y Agudelo, 2014; Martín, 2008; Visbal, Mendoza y García, 2015.

En ese contexto, la investigación tiene el objetivo de determinar los indices de eficiencia técnica de los años 2014 y 2015 a fin de realizar un análisis comparativo e identificar sus causas en las Carreras relacionadas con las Ciencias Económicas y Empresariales de las Universidades Públicas Autónomas del Estado Plurinacional de Bolivia a través de la aplicación del Análisis Envolvente de Datos (DEA) como una herramienta de análisis de la eficiencia técnica. Se plantea como hipótesis de trabajo: H1. Existen carreras con altos índices de eficiencia técnica. H2. En promedio existe un incremento de la eficiencia técnica entre el año 2014 y 2015; H3. El número de alumnos, titulados y docentes son las determinan los índices altos de eficiencia técnica en las carreras.

\section{Metodología}

La investigación es cuantitativa, tiene un enfoque aplicado y es de tipo empírico, como método considera la aplicación del modelo Análisis Envolvente de Datos (DEA) aplicadas a las carreras públicas relacionadas con el conocimiento de las Ciencias Económicas y Empresariales del Sistema de Educación Superior de Bolivia tanto en las centrales (ciudades) como en las sedes (provincia). Siendo la población total de 69 carreras en 11 facultades (ver tabla 1.) sobre la base de datos proporcionados por el Comité Ejecutivo de la Universidad Boliviana (CEUB) correspondiente a las gestiones académicas año 2014 y 2015 (CEUB, Boletín Estadístico, 2015) 
de las 11 Universidades Autónomas del SUB.Se determinan los índices de eficiencia correspondiente a las gestiones 2014- 2015.

Tabla 1. Población en función al grado de hhomogeneidad universitaria del Sistema Universitario Boliviano

\begin{tabular}{|l|l|l|l|}
\hline $\mathbf{N}^{\circ}$ & Universidades & Facultades & $\begin{array}{l}\text { Carreras } \\
\text { (Licenciatura) }\end{array}$ \\
\hline 1 & $\begin{array}{l}\text { Universidad Mayor, Real y Pontificia San Francisco Xavier } \\
\text { UMSFX }\end{array}$ & 1 & 7 \\
\hline 2 & Universidad Mayor de San Andrés UMSA & 1 & 6 \\
\hline 3 & Universidad Mayor de San Simón UMSS & 1 & 4 \\
\hline 4 & Universidad Autónoma Gabriel René Moreno UAGRM & 1 & 14 \\
\hline 5 & Universidad Autónoma Tomás Frías UATF & 1 & 10 \\
\hline 6 & Universidad Técnica de Oruro UTO & 1 & 4 \\
\hline 7 & Universidad Autónoma Juan Misael Saracho UAJMS & 1 & 7 \\
\hline 8 & Universidad Autónoma del Beni José Ballivián UABJB & 1 & 6 \\
\hline 9 & Universidad Nacional de Siglo XX UNSXX & 1 & 1 \\
\hline 10 & Universidad Amazónica de Pando UAP & 1 & 4 \\
\hline 11 & Universidad Pública de El Alto UPEA & 1 & \multicolumn{2}{|c|}{6} \\
\hline & TOTAL & $\mathbf{1 1}$ & $\mathbf{6 9}$ \\
\hline
\end{tabular}

Fuente: Elaboración propia, en base a datos del CEUB 2014 - 2015

El modelo DEA comprende el análisis de la eficiencia técnica (ET) (Farrell, 1957) con rendimientos de escala constantes (CCR) (Charnes, Cooper y Rhodes, 1978) y rendimientos variables (BCC) (Banker, Charnes y Cooper,1984). Asi, la función de producción universitaria considera los imputs y outputs propuesto por Ayaviri \& Zamora (2016, pág. 15) como también de: Visbal-Cadavid, Mendoza-Mendoza, \& Corredor-Carrascal, 2015); (Larrán-Jorge \& García-Correas, 2014); (Rolando, Delimiro, \& José Mario, 2015); (Amariles \& Soto-Mejía, 2015); (Cáceres V., Kristjanpoller R., \& Tabilo A., 2014); (Martín, 2008); (Ramírez \& Alfaro, 2013); (Coria, 2011); (Vásquez-Rojas, 2011) y (García-Correas \& Larrán-Jorge) quienes plantean inputs relacionados a los recursos económicos, docente en general y como outputs, investigaciones, titulaciones, inscripciones, entre otros. Asimismo la selección de los inputs y outputs cumplen una de las características más importante como es la homogeneidad existentes en las carreras relacionadas con el área del conocimiento de ciencias empresariales, se considera el modelo no paramétrico Análisis Envolvente de Datos (DEA), como mejor método a utilizar en la medición de la eficiencia universitaria como menciona Ayaviri \& Zamora (2016, pág. 18) donde plantean variables para la medición de la eficiencia universitaria en las universidades del Sistema Universitario Boliviano, como input: el número de docentes y como output: el número de graduados, número de investigaciones, número de participación en eventos científicos y producción científica que pueden ser aplicadas en la medición de la eficiencia universitaria, al margen de esa propuesta no se puede encontrar otros estudios al respecto. 
Bajo estas propuestas de función de producciones mencionadas en el cuadro 2, en la presente investigación también se considera la normativa del Sistema Universitario, siendo los inputs y outputs utilizados en la aplicación del Modelo DEA en el siguiente cuadro 3.

Cuadro 3. Identificación de Inputs y Outputs

\begin{tabular}{|c|c|}
\hline Inputs & Outputs \\
\hline Número Total de docente (NTD) & Número Total de Matriculados (NTM) \\
\hline $\begin{array}{c}\text { Docentes Tiempo Completo y Docentes } \\
\text { Tiempo Horario (Hombres, Mujeres y } \\
\text { Total) }\end{array}$ & Hombres, Mujeres y Total \\
\hline $\begin{array}{c}\text { Número Total de trabajadores } \\
\text { administrativos (NTA) }\end{array}$ & Número Total de Titulados (NTT) \\
\hline $\begin{array}{c}\text { Permanentes y eventuales (Hombres, } \\
\text { Mujeres y Total) }\end{array}$ & Hombres, Mujeres y Total \\
\cline { 2 - 2 } & Número Total de Matrícula Nueva (NTMN) \\
\cline { 2 - 2 } & Hombres, Mujeres y Total \\
\hline
\end{tabular}

Fuente: Elaboración propia, en base a datos del CEUB 2014 - 2015

Siendo la función de producción universitaria:

$\operatorname{Max} N T D, N T A(E)=f(N T M, N T T, N T M N)(i)$

$E=f(i)$

Dónde:

$\mathrm{NTD}=$ Número total de Docentes; considera tanto tiempo completo como tiempo horario; los cuales están dedicados a dar el valor agregado en la academia; transmisor de conocimientos a los universitarios.

NTA $=\quad$ Número total de Trabajadores Administrativos; tanto permanentes como eventuales; es el personal dedicado a la gestión institucional al interior de una universidad; responsable de los procesos administrativos. $\mathrm{NTM}=$ Número total de matriculados; universitarios que se registraron para proseguir estudios universitarios de continuidad; incluye a los nuevos universitarios; así como los que están en carrera.

NTT $=$ Número total de titulados; universitarios que lograron graduarse de una determinada carrera.

NTMN = Número total de matrícula nueva; registro de universitarios nuevos; los que ingresaron por primera vez a la universidad 


\section{Resultados}

\section{La Universidad en el Estado Plurinacional de Bolivia}

La universidad Boliviana se rige a una Aautonomía Universitaria como menciona la Constitución Política del Estado Plurinacional de Bolivia (2008), donde en su artículo 92 menciona que “(...) las Universidades Públicas son autónomas e iguales en jerarquía. La autonomía consiste en la libre administración de sus recursos; el nombramiento a sus autoridades, su personal docente y administrativo (...) ", asimismo,se rigen por el Estatuto Orgánico del Sistema de la Universidad Boliviana y se sustenta en los principios fundamentales de la Autonomía y el Cogobierno Paritario Docente - Estudiantil. La base de su organización descansa en la decisión soberana de docentes y estudiantes de la Universidad Pública Boliviana; expresada mediante el voto universal y la representación paritaria., tal como se manifiesta en el Artículo 2. (CEUB C. E., Estatuto Orgánico de la Universidad Boliviana, 2013).

El Modelo Académico es una representación ideal de referencia que se concreta en una estructura sistemática de funcionamiento que emana de los principios, fines y objetivos que ordena, regula e integra las funciones sustantivas del Sistema de la Universidad Boliviana para la formación integral de profesionales en cumplimiento del encargo social, para contribuir al desarrollo del Estado Plurinacional (Modelo Académico, CEUB, 2014, pág. 22). Los objetivos institucionales de la Universidad Boliviana regulada por la CEUB, vienen estipuladas en el Plan Nacional de Desarrollo Universitario 2014 - 2018 articulado con el Modelo Académico del Sistema de la Universidad Boliviana 2015 - 2019; donde ambos documentos hacen mención a las áreas estrategias que constituyen las dimensiones o pilares fundamentales en los que la Universidad Boliviana invertirá mayor atención y recursos (ver cuadro 4) donde cada una de las áreas estratégicas; tienen sus políticas; que son las decisiones programadas que guiarán el accionar del Sistema de la Universidad Boliviana; que permitirá alcanzar su misión y su visión del SUB, lo que significa que los indicadores cuantitativos y cualitativos determinan el logro de los objetivos estratégicos del SUB que son 217 metas operativas que plantean acciones específicas que permiten el desarrollo de las estrategias. (CEUB C. E., Plan Nacional de Desarrollo Universitario 2014 - 2018, 2014); en concreto lograr la calidad educativa de las universidades del SUB. 
Cuadro 4. Áreas estratégicas; del Plan de Desarrollo Universitario del Sistema de Universidades de Bolivia $2014-2018$

\begin{tabular}{|c|c|}
\hline ÀREA ESTRATÉGICA & OBJETIVO \\
\hline $\begin{array}{c}\text { Gestión de la Formación } \\
\text { Profesional de grado y } \\
\text { postgrado }\end{array}$ & $\begin{array}{l}\text { Sustentada en la necesidad de mejorar la calidad de la oferta } \\
\text { académica con currículos actualizados y potencial humano } \\
\text { altamente calificado, acreditada internacionalmente, que } \\
\text { garantice la formación de profesionales competitivos, } \\
\text { generadores de conocimientos que contribuyan al desarrollo } \\
\text { de las regiones y el país. En el caso de Posgrado, el área } \\
\text { estratégica está sustentada en la necesidad de mejorar las } \\
\text { capacidades de los profesionales de grado, a través de la } \\
\text { oferta de cursos de Posgrado de alto nivel competitivo. }\end{array}$ \\
\hline $\begin{array}{c}\text { Gestión de la Investigación, } \\
\text { ciencia, tecnología e } \\
\text { innovación }\end{array}$ & $\begin{array}{c}\text { Sustentada en la necesidad de que el Sistema de la } \\
\text { Universidad Boliviana, genere conocimiento a través de } \\
\text { procesos de investigación, desarrollo tecnológico e } \\
\text { innovación, para un aporte inmediato y permanente al } \\
\text { desarrollo sostenible del país }\end{array}$ \\
\hline $\begin{array}{l}\text { Gestión de la interacción } \\
\text { social - extensión } \\
\text { universitaria }\end{array}$ & $\begin{array}{c}\text { Gestión de la Interacción social - extensión universitaria. } \\
\text { Sustentada en la necesidad de que el Sistema de la } \\
\text { Universidad Boliviana se vincule efectivamente con su } \\
\text { entorno y que el conocimiento científico sea puesto a } \\
\text { disposición de la comunidad para contribuir a su desarrollo } \\
\text { integral sostenible. }\end{array}$ \\
\hline Gestión institucional & $\begin{array}{l}\text { Sustentada en la necesidad de lograr la conducción y soporte } \\
\text { necesarios para un óptimo desarrollo de las actividades } \\
\text { académicas de Formación Profesional, Investigación, } \\
\text { Ciencia, Tecnología e Innovación y de Interacción Social - } \\
\text { Extensión Universitaria }\end{array}$ \\
\hline
\end{tabular}

Fuente: Elaboración propia en base al Plan Nacional de Desarrollo Universitario 2014 2018 del CEUB y al Modelo Académico del Sistema de la Universidad Boliviana 2015 2019

El Sistema de la Universidad Boliviana categoriza a las universidades en:1) Universidades Autónomas; once (UMSFX, UMSA, UMSS, UAGRM, UATF, UTO, UAJMS, USBJB, UNSXX, UAP y UPEA), perciben ingresos por parte de Tesoro General de la Nación (TGN); Coparticipación Tributaria (CT), Impuesto Directo a los Hidrocarburos (IDH) y recursos propios (RP); 2)Universidades de Régimen Especial; tres; como: Universidad Católica Boliviana, Escuela Militar de Ingeniería y la Universidad Policial; y 3) Universidades de Régimen Especial de Postgrado; en la que se encuentra la Universidad Andina Simón Bolívar (CEUB, Modelo Académico del Sistema de la Universidad Boliviana, 2014, pág 18).

\section{Estudiantes, docentes y administrativos en la Universidad Publica en Bolivia}

Siendo importante para cada universidad el número de alumnos, docentes y administrativos para su funcionamiento, los datos universitarios 
muestran (ver tabla 2) que existe un crecimiento vegetativo del número de alumnos matriculados entre el año 2014 de un total de 403.198 estudiantes y en 2015 de un total de 417.220 estudiantes. Según la Secretaría Nacional de Desarrollo Institucional (2016, pág. 1), el crecimiento de la matrícula total alcanza a un 71,67\% en 12 años y del año 2004 al 2015 existe una tasa de crecimiento anual promedio del 5,97\%.

Los datos muestran que las Universidades UAGRM. UMSA, UMSS y UMSFX; son las cuatro universidades con mayor cantidad de universitarios superando los 50.000 universitarios matriculados; siendo la de mayor cantidad de estudiantes la UAGRM con 79.782 y la de mayor cantidad de matrícula nueva la UMSS con 14.760 nuevos estudiantes/año; además estas universidades tienen un ingreso de nuevos estudiantes por encima de los 8.000 universitarios/año; así también la cantidad de docentes supera los 1200 y en relación al personal administrativo es superior a 1100; categorizándose como grandes universidades (ver tabla 2).

Tabla 2. Universitarios Matriculados, Docentes y

Administrativos de las gestiones 2014 y 2015 del SUB

\begin{tabular}{|c|c|c|c|c|c|c|c|c|c|}
\hline \multirow[t]{2}{*}{$\mathrm{N}^{\circ}$} & \multirow[t]{2}{*}{$\begin{array}{l}\text { Universid } \\
\text { ades }\end{array}$} & \multicolumn{2}{|c|}{$\begin{array}{c}\text { MATRICULAD } \\
\text { OS }\end{array}$} & \multicolumn{2}{|c|}{$\begin{array}{c}\text { MATRICULA } \\
\text { NUEVA }\end{array}$} & \multicolumn{2}{|c|}{ DOCENTES } & \multicolumn{2}{|c|}{$\begin{array}{c}\text { ADMINISTRATI } \\
\text { VOS }\end{array}$} \\
\hline & & 2.014 & 2.015 & 2.014 & 2.015 & 2.014 & 2.015 & 2.014 & 2.015 \\
\hline 1 & UMSFX & 48.859 & 50.052 & 10.595 & 11.054 & 1.299 & 1.357 & 1.296 & 1.378 \\
\hline 2 & UMSA & 77.457 & 78.219 & 8.406 & 8.544 & 2.158 & 2.257 & 1.349 & 1.384 \\
\hline 3 & UMSS & 68.582 & 68.983 & 13.410 & 14.760 & 1.727 & 1.703 & 1.120 & 1.130 \\
\hline 4 & UAGRM & 78.941 & 79.782 & 12.484 & 13.072 & 1.728 & 1.750 & 1.670 & 1.809 \\
\hline 5 & UATF & 20.596 & 21.180 & 4.162 & 4.006 & 675 & 667 & 441 & 451 \\
\hline 6 & UTO & 23.523 & 24.377 & 3.700 & 3.641 & 732 & 783 & 645 & 663 \\
\hline 7 & UAJMS & 21.961 & 23.054 & 4.842 & 5.057 & 883 & 899 & 753 & 749 \\
\hline 8 & UABJB & 18.318 & 19.092 & 4.166 & 4.005 & 986 & 1.038 & 1.081 & 1.118 \\
\hline 9 & UNSXX & 7.764 & 7.904 & 2.742 & 2.861 & 352 & 367 & 159 & 178 \\
\hline 10 & UAP & 5.095 & 5.884 & 959 & 1.945 & 317 & 410 & 210 & 228 \\
\hline 11 & UPEA & 32.102 & 38.693 & 8.174 & 5.011 & 2.369 & 2.633 & 440 & 596 \\
\hline & TOTAL & $\begin{array}{c}403.19 \\
8\end{array}$ & $\begin{array}{c}417.22 \\
0\end{array}$ & 73.640 & 73.956 & 13.226 & 13.864 & 9.164 & 9.684 \\
\hline
\end{tabular}

Fuente: Elaboración propia en base a datos del Boletín $\mathrm{N}^{0} 5$ de la Secretaría Nacional de Desarrollo Institucional, del CEUB, 2016) 
Asimismo, se observa que las Universidades UAGRM. UMSA, UMSS y UMSFX; son las cuatro universidades con mayor cantidad de universitarios superando los 50.000 universitarios matriculados; siendo la de mayor cantidad de estudiantes la UAGRM con 79.782 y la de mayor cantidad de matrícula nueva la UMSS con 14.760 nuevos estudiantes/año; además estas universidades tienen un ingreso de nuevos estudiantes por encima de los 8.000 universitarios/año; así también la cantidad de docentes supera los 1200 y en relación al personal administrativo es superior a 1100; categorizándose como grandes universidades (ver tabla 2).

Mientras que la UPEA, UTO, UATF, UAJMS y UABJB; estás cinco universidades cuenta con universitarios entre 19.092 a los 38.693 universitarios matriculados; por consiguiente la de mayor cantidad de estudiantes es la UPEA con 38.693 matriculados y la de mayor cantidad de matrícula nueva la UAJMS con 5.057 nuevos estudiantes/año; en concreto estas universidades tienen un ingreso de nuevos estudiantes por encima de los 5.000 universitarios/año; así también la cantidad de docentes está entre los 667 a 1038 docentes y en relación al personal administrativo está entre los 451 a 1.118 docentes; categorizándose como medianas universidades. Sin embargo la UPEA presenta datos superiores con la mayor cantidad de docentes a nivel nacional, que alcanza a 2.633; cifra incluso superiores a las grandes universidades como son UAGRM; que sólo cuenta con 1750 docentes (ver tabla 2).

En esta misma línea la UNSXX y UAP estás dos universidades cuenta con universitarios entre 5.884 a los 7.904 universitarios matriculados; por otra parte la de mayor cantidad de estudiantes es la UNSXX con 7.904 matriculados y también la de mayor cantidad de matrícula nueva con 2.861 nuevos estudiantes/año; en concreto estas universidades tienen un ingreso de nuevos estudiantes por encima de los 1.900 universitarios/año; así también la cantidad de docentes está entre los 367 a 410 docentes y en relación al personal administrativo está entre los 178 a 228 docentes; categorizándose como pequeñas (ver tabla 2).

\section{Oferta académica en el Sistema Universitario}

La oferta académica de las carreras relacionadas con la ciencias empresariales muestra que existe en total una oferta académica de 422 carreras que incluye tanto las ciudades como las sedes desconcentradas, que abarcan a la mayoría de los municipios en el área de influencia de las universidades. (Secretaría Nacional de Desarrollo Institucional, 2016, pág. 2) (ver tabla 3) 
Tabla 3. Oferta Académica de las Universidades del SUB (Principales)

\begin{tabular}{|c|c|c|}
\hline $\mathrm{N}^{\mathbf{0}}$ & CARRERAS & MATRICULADOS 2015 \\
\hline 1 & Derecho & 47.963 \\
\hline 2 & Contaduría Pública & 37.909 \\
\hline 3 & Ingeniería Civil & 23.722 \\
\hline 4 & Administración de Empresas & 23.598 \\
\hline 5 & Medicina & 19.817 \\
\hline 6 & Ing. Comercial & 15.721 \\
\hline 7 & Enfermería & 12.437 \\
\hline 8 & Arquitectura & 10.564 \\
\hline 9 & Economía & 10.141 \\
\hline 10 & Ciencias de la Educación & 10.137 \\
\hline 11 & Ing. Industrial & 9.120 \\
\hline 12 & Psicología & 9.103 \\
\hline 13 & Odontología & 8.438 \\
\hline 14 & Ing. Agronómica & 7.718 \\
\hline 15 & Lic. en Trabajo Social & 6.626 \\
\hline 16 & Ing. de Sistemas & 6.603 \\
\hline 17 & Ing. Financiera & 5.743 \\
\hline 18 & Ing. Química & 5.474 \\
\hline 19 & Ing. Electromecánica & 5.195 \\
\hline 20 & Ing. Petrolera & 4.686 \\
\hline 21 & Ciencias de la Comunicación Social & 4.604 \\
\hline 22 & Ing. en Sistemas & 4.529 \\
\hline 23 & Medicina, Veterinaria y Zootecnia & 5.410 \\
\hline 24 & Bioquímica y Farmacia & 4.156 \\
\hline & Eacion propa en base & \\
\hline
\end{tabular}

Fuente: Elaboración propia en base a datos del Boletín $\mathrm{N}^{\circ} 5$ de la Secretaría

Nacional de Desarrollo Institucional, del CEUB, 2016), Pág. 14

\section{Aplicación del Modelo DEA en las carreras de las Ciencias Económicas y Empresariales}

Los resultados de la aplicación del modelo DEA a las carreras del area de conocimiento de ciencias económicas e empresariales en las Universidades Bolivianas pertenecientes al Sistema Público muestran indices que permiten responder a la preguntas icuales son los indices de eficiencia tecnica?. Y demostrar que H1. Existen carreras en el área de conocimiento de las Ciencias Económicas y Empresariales con altos índices de eficiencia técnica y H2. En promedio existe un incremento de la eficiencia técnica entre el año 2014 y 201.

Los índices de eficiencia muestran que alcanzan el $100 \%$ tanto del modelo CCR y BCC la Carrera de Administración de Empresas de la Universidad Mayor de San Simón, Carrera de Economía de la Universidad Autónoma Tomas Frías y la Carrera de Administración de Empresas de la Universidad Técnica de Oruro; el resto de las carreras en las Ciencias 
Económicas y Empresariales se encuentran por debajo del 100\%, lo que significa que deben trabajar en la mejora de estos índices (ver tabla 4).

Tabla 4. Índice de eficiencia técnica de las Carreras de Ciencias Económicas y Empresariales

\begin{tabular}{|c|c|c|c|c|c|c|c|c|c|}
\hline $\mathbf{N}^{\circ}$ & CARRERAS & $\mathbf{U}$ & $\mathbf{F}$ & $\begin{array}{l}\text { CCR } \\
2014 \\
\end{array}$ & $\begin{array}{l}\text { CCR } \\
2015 \\
\end{array}$ & $\begin{array}{l}\text { BCC } \\
2014 \\
\end{array}$ & $\begin{array}{l}\text { BCC } \\
2015 \\
\end{array}$ & $\begin{array}{l}\text { EE } \\
2014 \\
\end{array}$ & EE 2015 \\
\hline 1 & ADMINISTRACIÓN DE EMPRESAS & 1 & 2 & 78,59 & 100 & 100 & 100 & 78,59 & 100,00 \\
\hline 2 & ADMINISTRACIÓN FINANCIERA & 1 & 2 & 56,9 & 66,93 & 65,34 & 67,25 & 87,08 & 99,52 \\
\hline 3 & CONTADURÍA PÚBLICA & 1 & 2 & 53,68 & 65,31 & 59,77 & 65,33 & 89,81 & 99,97 \\
\hline 4 & INGENIERÍA COMERCIAL & 1 & 2 & 45,86 & 80,62 & 76,6 & 90,6 & 59,87 & 88,98 \\
\hline 5 & GESTIÓN Y GERENCIA DE NEGOCIOS & 1 & 2 & 36,21 & 70,44 & 36,21 & 70,43 & 100,00 & 100,0 \\
\hline 6 & ECONOMÍA & 1 & 2 & 30,4 & 44,25 & 34,72 & 44,28 & 87,56 & 99,93 \\
\hline 7 & GESTIÓN PÚBLICA & 1 & 2 & 22,68 & 32,55 & 23,59 & 34,06 & 96,14 & 95,57 \\
\hline 8 & CONTADURÍA PÚBLICA & 2 & 2 & 91,56 & 100 & 100 & 100 & 91,56 & 100,00 \\
\hline 9 & ECONOMÍA & 2 & 2 & 42,44 & 29,59 & 59,66 & 57,77 & 71,14 & 51,22 \\
\hline 10 & ADMINISTRACIÓN DE EMPRESAS & 2 & 2 & 34,85 & 56,47 & 63,46 & 67,34 & 54,92 & 83,86 \\
\hline 11 & TURISMO & 2 & 2 & 28 & 29,25 & 39,36 & 35,2 & 71,14 & 83,10 \\
\hline 12 & HISTORIA & 2 & 2 & 14,01 & 13,17 & 19,28 & 16,79 & 72,67 & 78,44 \\
\hline 13 & ESTADÍSTICA & 2 & 2 & 6,01 & 7 & 7,98 & 7,67 & 75,31 & 91,26 \\
\hline 14 & ADMINISTRACIÓN DE EMPRESAS & 3 & 2 & 100 & 100 & 100 & 100 & 100,00 & 100,00 \\
\hline 15 & ECONOMÍA & 3 & 2 & 44,94 & 41,54 & 48,61 & 41,58 & 92,45 & 99,90 \\
\hline 16 & ADMINISTRACIÓN DE EMPRESAS & 3 & 2 & 42,65 & 37,32 & 91,15 & 77,35 & 46,79 & 48,25 \\
\hline 17 & CONTADURÍA PÚBLICA & 3 & 2 & 39,8 & 50,58 & 100 & 100 & 39,80 & 50,58 \\
\hline 18 & GESTIÓN DE TURISMO & 4 & 2 & 83,79 & 64,7 & 100 & 93,91 & 83,79 & 68,90 \\
\hline 19 & GESTIÓN DE TURISMO & 4 & 2 & 79,52 & 100 & 100 & 100 & 79,52 & 100,00 \\
\hline 20 & CONTADURÍA PÚBLICA & 4 & 2 & 67,19 & 55,08 & 85,89 & 56,24 & 78,23 & 97,94 \\
\hline 21 & ADMINISTRACIÓN DE EMPRESAS & 4 & 2 & 53,17 & 94,29 & 100 & 100 & 53,17 & 94,29 \\
\hline 22 & CONTABILIDAD & 4 & 2 & 48,93 & 72,15 & 53,54 & 72,22 & 91,39 & 99,90 \\
\hline 23 & CONTADURÍA PÚBLICA & 4 & 2 & 45,15 & 53,19 & 46,65 & 53,41 & 96,78 & 99,59 \\
\hline 24 & ADMINISTRACIÓN DE EMPRESAS & 4 & 2 & 30,84 & 35,86 & 68,22 & 67,89 & 45,21 & 52,82 \\
\hline 25 & INGENIERÍA FINANCIERA & 4 & 2 & 28,42 & 39,08 & 68,59 & 65,62 & 41,43 & 59,56 \\
\hline 26 & INGENIERÍA COMERCIAL & 4 & 2 & 22,14 & 39,51 & 63,68 & 66,84 & 34,77 & 59,11 \\
\hline 27 & CONTADURÍA PÚBLICA & 4 & 2 & 19,15 & 36,17 & 100 & 100 & 19,15 & 36,17 \\
\hline 28 & INFORMACIÓN Y CONTROL DE GESTIÓN & 4 & 2 & 15,68 & 28,05 & 16,75 & 28,12 & 93,61 & 99,75 \\
\hline 29 & ECONOMÍA & 4 & 2 & 13,5 & 31,9 & 25,64 & 31,91 & 52,65 & 99,97 \\
\hline 30 & ADMINISTRACIÓN DE EMPRESAS & 4 & 2 & 11,12 & 33,33 & 11,88 & 33,44 & 93,60 & 99,67 \\
\hline 31 & CONTADURÍA PÚBLICA & 4 & 2 & 2,26 & 32,53 & 2,33 & 35,77 & 97,00 & 90,94 \\
\hline 32 & ECONOMÍA & 5 & 2 & 100 & 100 & 100 & 100 & 100,00 & 100,00 \\
\hline 33 & CONTADURÍA PÚBLICA & 5 & 2 & 55,35 & 64,28 & 58,12 & 65,48 & 95,23 & 98,17 \\
\hline 34 & CONTABILIDAD Y FINANZAS & 5 & 2 & 50,31 & 39,35 & 52,51 & 41,22 & 95,81 & 95,46 \\
\hline 35 & ECONOMÍA & 5 & 2 & 39,76 & 74,45 & 41,5 & 74,64 & 95,81 & 99,75 \\
\hline 36 & ESTADÍSTICA & 5 & 2 & 39,39 & 57,83 & 41,42 & 57,83 & 95,10 & 100,00 \\
\hline 37 & ADMINISTRACIÓN DE EMPRESAS & 5 & 2 & 36,2 & 35,85 & 46,93 & 44,94 & 77,14 & 79,77 \\
\hline 38 & TURISMO & 5 & 2 & 34,87 & 53,49 & 35,5 & 53,49 & 98,23 & 100,00 \\
\hline 39 & TURISMO & 5 & 2 & 34,52 & 47,48 & 35,4 & 50,86 & 97,51 & 93,35 \\
\hline 40 & CONTADURÍA PÚBLICA & 5 & 2 & 24,49 & 24,2 & 24,91 & 24,47 & 98,31 & 98,90 \\
\hline 41 & ECONOMÍA & 5 & 2 & 22,57 & 10,59 & 23,15 & 10,76 & 97,49 & 98,42 \\
\hline 42 & ADMINISTRACIÓN DE EMPRESAS & 6 & 2 & 100 & 100 & 100 & 100 & 100,00 & 100,00 \\
\hline 43 & CONTADURÍA PÚBLICA & 6 & 2 & 57 & 46,36 & 63,56 & 55,62 & 89,68 & 83,35 \\
\hline 44 & ECONOMÍA E INGENIERÍA COMERCIAL & 6 & 2 & 39,48 & 52,31 & 39,47 & 52,31 & 100,00 & 100,00 \\
\hline 45 & CONTADURÍA PÚBLICA & 6 & 2 & 10,4 & 7,48 & 11,34 & 7,56 & 91,71 & 98,94 \\
\hline 46 & COMERCIO INTERNACIONAL & 7 & 2 & 36,98 & 48,88 & 67,28 & 62,34 & 54,96 & 78,41 \\
\hline
\end{tabular}




\begin{tabular}{|l|l|l|l|l|l|l|l|l|l|}
47 & INGENIERÍA COMERCIAL & 7 & 2 & 36,52 & 28,85 & 37,58 & 28,91 & 97,18 & 99,79 \\
\hline 48 & CONTADURÍA PÚBLICA & 7 & 2 & 29,56 & 27,85 & 68,82 & 63,99 & 42,95 & 43,52 \\
\hline 49 & ECONOMÍA & 7 & 2 & 26,88 & 20 & 28,98 & 20,06 & 92,75 & 99,70 \\
\hline 50 & CONTADURÍA PÚBLICA & 7 & 2 & 22,63 & 35,97 & 28,89 & 36 & 78,33 & 99,92 \\
\hline 51 & ADMINISTRACIÓN DE EMPRESAS & 7 & 2 & 21,1 & 22,96 & 35,82 & 36,38 & 58,91 & 63,11 \\
\hline 52 & CONTADURÍA PÚBLICA & 7 & 2 & 17,35 & 34,27 & 17,85 & 34,35 & 97,20 & 99,77 \\
\hline 53 & CONTADURÍA PÚBLICA & 8 & 2 & 26,03 & 16,72 & 36,36 & 26,04 & 71,59 & 64,21 \\
\hline 54 & CONTADURÍA PÚBLICA & 8 & 2 & 18,91 & 12,72 & 35,15 & 25,79 & 53,80 & 49,32 \\
\hline 55 & ADMINISTRACIÓN DE EMPRESAS & 8 & 2 & 13 & 14,88 & 22,62 & 22,95 & 57,47 & 64,84 \\
\hline 56 & ADMINISTRACIÓN DE EMPRESAS & 8 & 2 & 12,84 & 9,74 & 15,42 & 14,08 & 83,27 & 69,18 \\
\hline 57 & ECONOMÍA & 8 & 2 & 9,74 & 11,48 & 11,08 & 11,58 & 87,91 & 99,14 \\
\hline 58 & TURISMO & 8 & 2 & 5,26 & 8,06 & 10,25 & 10,31 & 51,32 & 78,18 \\
\hline 59 & CONTADURÍA PÚBLICA & 9 & 2 & 77,99 & 63,76 & 98,84 & 76,59 & 78,91 & 83,25 \\
\hline 60 & ADMINISTRACIÓN DE EMPRESAS & 10 & 2 & 16,43 & 41,56 & 20,63 & 43,78 & 79,64 & 94,93 \\
\hline 61 & INGENIERÍA COMERCIAL & 10 & 2 & 14,83 & 18,2 & 15,68 & 18,6 & 94,58 & 97,85 \\
\hline 62 & CONTADURÍA PÚBLICA & 10 & 2 & 9,12 & 22,01 & 9,25 & 22,58 & 98,59 & 97,48 \\
\hline 63 & TURISMO & 10 & 2 & 8,88 & 10,08 & 9,11 & 10,56 & 97,48 & 95,45 \\
\hline 64 & COMERCIO INTERNACIONAL & 11 & 2 & 35,98 & 21,07 & 56,32 & 32,42 & 63,88 & 64,99 \\
\hline 65 & CONTADURÍA PÚBLICA & 11 & 2 & 26,49 & 28,08 & 84,31 & 91,9 & 31,42 & 30,55 \\
\hline 66 & ADMINISTRACIÓN DE EMPRESAS & 11 & 2 & 17,83 & 22,25 & 42,18 & 54,64 & 42,27 & 40,72 \\
\hline 67 & GESTIÓN TURISTICA Y HOTELERA & 11 & 2 & 16,73 & 17,24 & 26,78 & 27 & 62,47 & 63,85 \\
\hline 68 & ECONOMÍA & 11 & 2 & 10,06 & 9,89 & 19,38 & 20,17 & 51,91 & 49,03 \\
\hline 69 & HISTORIA & 11 & 2 & 4,63 & 4,57 & 6,89 & 6,96 & 67,20 & 65,66 \\
\hline & PROMEDIO & & & $\mathbf{3 5 , 5}$ & $\mathbf{4 2 , 1}$ & $\mathbf{4 8 , 5}$ & $\mathbf{5 1 , 0}$ & $\mathbf{7 6 , 9}$ & $\mathbf{8 3 , 6}$ \\
\hline
\end{tabular}

Fuente: Elaboración propia

Asimismo se puede identificar que en la mayoria de las carreras relacionadas con las Ciencias Económicas y Empresariales existe un incremento entre la eficiencia tecnica del año 2014 y 2015. Lo que signfica que existen elementos importantes en las variables que definen los indices de eficiencia y dependiendo de ellas se podra observar si existe un incremento o disminución.

\section{Relación de los indices de eficiencia técnica con el numero de estudiantes}

Los resultados de eficiencia, muestran que H3. El número de alumnos matriculados y titulados son las que determinan los índices altos de eficiencia técnica en las carreras relacionadas con el área del conocimiento de Ciencias Económicas y Empresariales, porque las carreras en las universidades con mayor cantidad de universitarios matriculados son más eficientes; en relación a las carreras de las universidades de menor cantidad de matriculados. La tabla 5, muestra que la eficiencia global promedio para el año 2014 alcanza el 35,5\% y el año 2015 un 42,1 \% con relación al modelo CCR y con el modelo BCC alcanza para el año 2014 una eficienica global de 48,5 \% y para el año 2015 51\%, donde la eficiencia de escala alcanza para el año 2014 el 76,9\% y para el año 2015 el 83,6\% (ver tabla 5). El incremento de la eficiencia técnica global de 2015 respecto a 
2014 no puede atribuirse a la eficiencia de escala, por lo que la facultades pasaron a producir por debajo de la escala óptima.

Tabla 5. Índices de eficiencia de las carreas y su relación con el número de estudiantes y titulados

\begin{tabular}{|c|c|c|c|c|c|c|c|}
\hline $\mathrm{N}^{\mathrm{o}}$ & TITULO DE CARRERA & $\mathbf{U}$ & $\begin{array}{c}\text { MEDIA } \\
\text { MATRICULA }\end{array}$ & $\begin{array}{c}\text { MEDIA } \\
\text { TITULADOS }\end{array}$ & $\begin{array}{c}\text { MEDIA } \\
\text { CCR }\end{array}$ & MEDIABCC & $\begin{array}{l}\text { MEDIA } \\
\text { EE }\end{array}$ \\
\hline 1 & Lic. en Administración de Empresas & 1 & 2683,00 & 255,50 & 89,30 & 100,00 & 93,53 \\
\hline 2 & Lic. en Administración de Empresas & 2 & 3670,50 & 266,00 & 45,66 & 65,40 & 30,99 \\
\hline 3 & Lic. en Administración de Empresas & 3 & 3721,50 & 300,00 & 39,99 & 84,25 & 64,44 \\
\hline 4 & Ing. Comercial & 3 & 2997,00 & 64,00 & 100,00 & 100,00 & 96,22 \\
\hline 5 & Lic. en Administración de Empresas & 4 & 3536,00 & 131,50 & 33,35 & 68,06 & 96,47 \\
\hline 6 & Lic. en Administración de Empresas & 4 & 355,00 & 17,00 & 22,23 & 22,66 & 89,76 \\
\hline 7 & Lic. en Administración de Empresas & 4 & 352,50 & 9,50 & 73,73 & 100,00 & 47,52 \\
\hline 8 & Lic. en Administración de Empresas & 5 & 1583,00 & 52,00 & 36,03 & 45,94 & 61,01 \\
\hline 9 & Lic. en Administración de Empresas & 6 & 1006,00 & 81,50 & 100,00 & 100,00 & 86,52 \\
\hline 10 & Lic. en Administración de Empresas & 7 & 1577,50 & 105,50 & 22,03 & 36,10 & 98,49 \\
\hline 11 & Lic. en Administración de Empresas & 8 & 664,50 & 24,00 & 13,94 & 22,79 & 93,97 \\
\hline 12 & Lic. en Administración de Empresas & 8 & 303,00 & 0,00 & 11,29 & 14,75 & 100,00 \\
\hline 13 & Lic. en Administración de Empresas & 10 & 636,00 & 34,50 & 29,00 & 32,21 & 96,70 \\
\hline 14 & Lic. en Administración de Empresas & 11 & 1608,50 & 3,50 & 20,04 & 48,41 & 67,90 \\
\hline 15 & Lic. en Administración Financiera & 1 & 1591,00 & 133,00 & 61,92 & 66,30 & 89,13 \\
\hline 16 & Lic. en Comercio Internacional & 7 & 252,00 & 18,50 & 42,93 & 64,81 & 75,56 \\
\hline 17 & Lic. en Comercio Internacional & 11 & 637,50 & 0,00 & 28,53 & 44,37 & 97,55 \\
\hline 18 & Lic. en Contaduría Pública & 4 & 630,00 & 20,00 & 60,54 & 62,88 & 96,64 \\
\hline 19 & Lic. en Contabilidad y Finanzas & 5 & 1288,50 & 51,50 & 44,83 & 46,87 & 98,49 \\
\hline 20 & Lic. en Contaduría Pública & 1 & 2204,50 & 187,00 & 59,50 & 62,55 & 61,16 \\
\hline 21 & Lic. en Contaduría Pública & 2 & 5758,50 & 641,50 & 95,78 & 100,00 & 63,16 \\
\hline 22 & Lic. en Contaduría Pública & 3 & 5925,00 & 221,00 & 45,19 & 100,00 & 50,47 \\
\hline 23 & Lic. en Contaduría Pública & 4 & 6065,50 & 243,50 & 27,66 & 100,00 & 66,43 \\
\hline 24 & Lic. en Contaduría Pública & 4 & 903,50 & 20,50 & 49,17 & 50,03 & 95,43 \\
\hline 25 & Lic. en Contaduría Pública & 4 & 249,00 & 6,00 & 61,14 & 71,07 & 77,12 \\
\hline 26 & Lic. en Contaduría Pública & 4 & 33,50 & 11,00 & 17,40 & 19,05 & 89,30 \\
\hline 27 & Lic. en Contaduría Pública & 5 & 1470,50 & 78,00 & 59,82 & 61,80 & 96,23 \\
\hline 28 & Lic. en Contaduría Pública & 5 & 594,50 & 6,00 & 24,35 & 24,69 & 76,31 \\
\hline 29 & Lic. en Contaduría Pública & 6 & 2358,00 & 148,50 & 51,68 & 59,59 & 76,23 \\
\hline 30 & Lic. en Contaduría Pública & 6 & 68,50 & 0,00 & 8,94 & 9,45 & 93,30 \\
\hline 31 & Lic. en Contaduría Pública & 7 & 2818,00 & 252,00 & 28,71 & 66,41 & 64,75 \\
\hline 32 & Lic. en Contaduría Pública & 7 & 920,00 & 19,00 & 29,30 & 32,45 & 100,00 \\
\hline 33 & Lic. en Contaduría Pública & 7 & 463,00 & 26,50 & 25,81 & 26,10 & 50,50 \\
\hline 34 & Lic. en Contaduría Pública & 8 & 847,00 & 52,50 & 21,38 & 31,20 & 99,12 \\
\hline 35 & Lic. en Contaduría Pública & 8 & 822,50 & 0,00 & 15,82 & 30,47 & 78,46 \\
\hline 36 & Lic. en Contaduría Pública & 9 & 899,00 & 0,00 & 70,88 & 87,72 & 98,61 \\
\hline 37 & Lic. en Contaduría Pública & 10 & 312,00 & 11,50 & 15,57 & 15,92 & 45,19 \\
\hline 38 & Lic. en Contaduría Pública & 11 & 3044,50 & 27,50 & 27,29 & 88,11 & 98,04 \\
\hline 39 & Lic. en Economía & 1 & 1282,00 & 90,50 & 37,33 & 39,50 & 66,69 \\
\hline 40 & Lic. en Economía & 2 & 3171,50 & 177,50 & 36,02 & 58,72 & 81,08 \\
\hline 41 & Lic. en Economía & 3 & 1098,50 & 75,50 & 43,24 & 45,10 & 100,00 \\
\hline 42 & Lic. en Economía & 4 & 1008,50 & 49,50 & 22,70 & 28,78 & 95,33 \\
\hline 43 & Lic. en Economía & 5 & 926,50 & 70,00 & 57,11 & 58,07 & 97,96 \\
\hline 44 & Lic. en Economía & 5 & 296,00 & 8,50 & 16,58 & 16,96 & 83,29 \\
\hline 45 & Lic. en Economía & 5 & 231,00 & 0,50 & 100,00 & 100,00 & 61,18 \\
\hline 46 & Lic. en Economía & 7 & 488,50 & 40,00 & 23,44 & 24,52 & 95,65 \\
\hline
\end{tabular}




\begin{tabular}{|c|c|c|c|c|c|c|c|}
\hline 47 & Lic. en Economía & 8 & 338,50 & 20,00 & 10,61 & 11,33 & 96,18 \\
\hline 48 & Lic. en Economía & 11 & 646,50 & 11,00 & 9,98 & 19,78 & 95,64 \\
\hline 49 & Lic. en Economía & 6 & 364,00 & 10,50 & 45,90 & 45,89 & 76,35 \\
\hline 50 & Lic. en Estadística & 2 & 254,50 & 5,50 & 6,51 & 7,83 & 69,39 \\
\hline 51 & Lic. en Estadística & 5 & 138,50 & 2,50 & 48,61 & 49,63 & 93,75 \\
\hline 52 & Lic. en Gestión de Turismo & 4 & 697,00 & 20,50 & 89,76 & 100,00 & 97,78 \\
\hline 53 & Lic. en Filología Hispánica & 4 & 694,00 & 9,00 & 74,25 & 96,96 & 100,00 \\
\hline 54 & Lic. en Gestión Pública & 1 & 482,00 & 37,00 & 27,62 & 28,83 & 49,02 \\
\hline 55 & Lic. en Gestión Turística y Hotelera & 11 & 499,00 & 0,00 & 16,99 & 26,89 & 96,68 \\
\hline 56 & Lic. en Gestión y Gerencia de Negocios & 1 & 126,50 & 2,00 & 53,33 & 53,32 & 100,00 \\
\hline 57 & Lic. en Historia & 2 & 599,50 & 12,00 & 13,59 & 18,04 & 27,66 \\
\hline 58 & Lic. en Historia & 11 & 194,00 & 0,00 & 4,60 & 6,93 & 95,78 \\
\hline 59 & Lic. en Información y Control de Gestión & 4 & 455,50 & 19,00 & 21,87 & 22,44 & 73,73 \\
\hline 60 & $\begin{array}{l}\text { Ing. Comercial } \\
\end{array}$ & 1 & 2053,00 & 155,00 & 63,24 & 83,60 & 51,56 \\
\hline 61 & Ing. Comercial & 4 & 3835,00 & 153,50 & 30,83 & 65,26 & 41,50 \\
\hline 62 & Ing. Comercial & 7 & 556,50 & 33,50 & 32,69 & 33,25 & 46,94 \\
\hline 63 & Ing. Comercial & 10 & 461,00 & 8,50 & 16,52 & 17,14 & 98,19 \\
\hline 64 & Ing. Financiera & 4 & 3166,50 & 98,00 & 33,75 & 67,11 & 87,29 \\
\hline 65 & Lic. en Turismo & 2 & 1463,00 & 68,50 & 28,63 & 37,28 & 43,24 \\
\hline 66 & Lic. en Turismo & 5 & 217,00 & 32,50 & 41,00 & 43,13 & 95,86 \\
\hline 67 & Lic. en Turismo & 5 & 129,00 & 0,00 & 44,18 & 44,50 & 74,43 \\
\hline 68 & Lic. en Turismo & 8 & 386,50 & 7,50 & 6,66 & 10,28 & 88,09 \\
\hline 69 & Lic. en Turismo Sostenible & 10 & 133,00 & 5,00 & 9,48 & 9,84 & 94,89 \\
\hline & MEDIA & 11 & 1322,35 & 68,73 & $\mathbf{3 , 0 0}$ & 7,00 & $\mathbf{5 , 0 0}$ \\
\hline
\end{tabular}

Fuente: Elaboración propia

Asimismo, se observa que de 10 carreras eficientes , 3 son a traves del modelo de CCR, 7 BCC y 5 efiencia de escala.

\section{Discusion y conclusiones}

\section{¿Cuáles son las explicaciones de las ineficiencias en las carreras del area de conocimiento de Ciencias Economicas y Empresariales?}

De acuerdo con los resultados de los indices de eficiencia se puede identificar altos y bajos indices de eficiencia, sin embargo, es importante responder a una pregunta ¿ cuales son las causas?, en ese contexto, la investigación responde a traves de la busqueda de explicaciones a partir de la aplicación de la regresión lineal. Se procedió a considerar variables relacionadas con los alumnos, docentes y administrativos, a fin de determina si estas variables son las que explican (ver cuadro 5.) los indices de la tabla 4. . 
Cuadro 5. Variables explicativas de la eficienica técnica

\begin{tabular}{|c|c|c|c|}
\hline MODELO & VARIABLES & VARIABLES & VARIABLE \\
& INDEPENDIENTES & INDEPENDIENTES & DEPENDIENTE \\
& & (MOMENCLATURA EN & (INDICE DE \\
& & & EFICIENCIA E N \\
& & & LOS DOS \\
& & MODELOS) \\
\hline Modelo 1. & Número Total de Titulados, & a. Predictores: (Constante), & b. Variable \\
2014 Y 2015 & Número Total de & Administrativos Total 2014, & dependiente: \\
& Estudiantes Matriculados & Titulados Total 2014, & CCR2014, CCR2015 \\
& Número Total con & Matricula Total Año 2014, & BCC2014, BCC2015 \\
& Matricula Nueva, Número & Docentes Total 2014, & EE2014, EE2015 \\
& Total de Docentes, El & Matricula Nueva Total 2014. & \\
& Número Total de & & \\
& Administrativos que & & \\
& trabajan & & \\
\hline Modelo 2. & Número Total de Titulados, & a. Predictores: (Constante), & b. Variable \\
& Número Total de & Titulados Total 2014, & dependiente: \\
& Estudiantes Matriculados & Matricula Total Año 2014, & CCR2014, CCR2015 \\
& Número Total con & Matricula Nueva Total 2014. & BCC2014, BCC2015 \\
& Matricula Nueva, & & EE2014, EE2015 \\
\hline Modelo3. 2015 & Número Total de Docentes, & a. Predictores: (Constante), & b. Variable \\
& El Número Total de & Administrativos Total 2014, & dependiente: \\
& Administrativos que & Docentes Total 2014. & CCR2014, CCR2015 \\
& trabajan & BCC2014, BCC2015 \\
& & & EE2014, EE2015 \\
\hline
\end{tabular}

Fuente: Elaboración propia con base de datos CEUB, 2014 -2015. CCR (Eficiecia con rendimientos Crecientes), BCC (Eficiencia con redimientos constantes, EE(Eficiencia de escala)

Los resultados de la aplicación de regresión lineal consideradon las variables explicativas del cuadro 5. permite idendentificar que los factores causales para la eficiencia técnica universitaria en las carreras relacionadas con el conocimiento de la ciencias economicas y empresariales tanto para el año 2014 y 2015 vienen explicadas por aquellos factores,como: Número Total de Titulados, Número Total de Estudiantes Matriculados Número Total con Matricula Nueva,1 Número Total de Administrativos que trabajan , Número Total de Titulados (MODELO 2) (ver tabla 6), lo que signfica que se rechaza la hipotesis nula aceptandose que existe una relación lineal significativa entre las variables $\mathrm{X}$ y $\mathrm{Y}$, explicando estas variables en el modelo BCC en un 66,3\% en el año 2014 y un 66,8\% en el año 2015. Considerandose que su correlación es alta (ver tabla 6). 
Tabla 6. Resultados de la Regresión lineal bajo el modelo 1

\begin{tabular}{|c|c|c|c|c|c|c|c|c|c|}
\hline \multirow[b]{2}{*}{$\begin{array}{c}\text { AREA DE } \\
\text { CONOCIMIENTO }\end{array}$} & \multirow[b]{2}{*}{$\begin{array}{l}\text { b. Variable } \\
\text { dependiente }\end{array}$} & \multirow[b]{2}{*}{ Modelo } & \multirow[b]{2}{*}{$\mathrm{R}$} & \multirow[b]{2}{*}{$\begin{array}{c}\mathrm{R} \\
\text { cuadrado }\end{array}$} & \multirow[b]{2}{*}{$\begin{array}{c}\mathrm{R} \\
\text { cuadrado } \\
\text { ajustado }\end{array}$} & \multirow{2}{*}{$\begin{array}{c}\text { Error } \\
\text { estándar } \\
\text { de la } \\
\text { estimación }\end{array}$} & \multirow[b]{2}{*}{$\begin{array}{c}\text { Cambio } \\
\text { en F }\end{array}$} & \multirow[b]{2}{*}{$\begin{array}{c}\text { Sig. } \\
\text { Cambio } \\
\text { en F }\end{array}$} & \multirow[b]{2}{*}{$\begin{array}{l}\text { Durbin- } \\
\text { Watson }\end{array}$} \\
\hline & & & & & & & & & \\
\hline \multirow[t]{2}{*}{ ECONOMIA * } & CCR2014 & 1 &, $531^{\mathrm{a}}$ & ,282 &, 225 & 21,69 & 4,96 &, 001 & 2,187 \\
\hline & CCR2015 & 1 &, $662^{\mathrm{a}}$ & ,439 & ,394 & 20,96 & 9,85 & ,000 & 2,006 \\
\hline \multirow[t]{2}{*}{ ECONOMIA* } & BCC2014 & 2 &, $663^{\mathrm{a}}$ & ,439 & ,395 & 23,85 & 9,88 &, 000 & 2,271 \\
\hline & BCC2015 & 2 &, $668^{\mathrm{a}}$ & ,447 & ,403 & 22,42 & 10,17 & ,000 & 2,223 \\
\hline \multirow[t]{2}{*}{ ECONOMIA* } & EE 2014 & 3 &, $624^{\mathrm{a}}$ & ,390 & ,319 & 24,09 & 5,50 & ,001 & 1,602 \\
\hline & EE 2015 & 3 &, $436^{\mathrm{a}}$ &, 190 &, 096 & 19,11 & 2,02 &, 095 & 2,031 \\
\hline
\end{tabular}

\section{Fuente: Elaboracion propia}

- $\quad$ Corresponde a las carreras de Cienicas Economicas y Empresariales (ECONOMIA). Carreras que aparecen en la Tabla 5.

Concluyéndose que las variables que explican las ineficiencias en las carreras relacionadas con las Ciencias Económicas y Empresariales están relacionadas con factores, como: el Número Total de Titulados, Número Total de Estudiantes Matriculados Número Total con Matricula Nueva Número Total de Administrativos que trabajan, Número Total de Titulados. Puesto que se demuestra que la eficiencia técnica universitaria no depende del número de estudiantes que se tenga en las carreras, sino de otros factores.

\section{¿La selección de variables inputs y ouputs son pertinentes?}

En el cuadro 6. Se puede observar distintos autores que utilizan variables que en la investigación se utilizaron para medir la eficiencia técnica en las universidades con características similares a las Universidades Publicas del Estado Plurinacional de Bolivia. Ya que la selección de las variables se constituyen en la fase más importante del desarrollo de una evaluación mediante la técnica DEA y considerando que la validez y confiabilidad de los resultados obtenidos, dependen en gran medida de una adecuada elección de las variables. 


\section{Cuadro 6. Inputs y Outputs en relación a las investigaciones realizadas}

\begin{tabular}{|c|c|c|c|}
\hline Inputs & Investigaciones & Autores & $\begin{array}{c}\text { Cuántos estudios al } \\
\text { respecto para la variable } \\
\text { aplicada }\end{array}$ \\
\hline \multirow{7}{*}{$\begin{array}{l}\text { NÚMERO TOTAL DEDOCENTES } \\
\text { (NTD) Docentes Tiempo Completoy } \\
\text { Docentes Tiempo Horario (Hombres, } \\
\text { Mujeres y Total) }\end{array}$} & Docentes tiempo completo equivalente. & Amariles y Soto, 2015 & \multirow{7}{*}{$\begin{array}{l}\text { Varios estudios al respecto, } \\
\text { existe aproximación. }\end{array}$} \\
\hline & $\begin{array}{l}\text { Catedráticos universidad, catedráticos escuela y titulares } \\
\text { universidad }\end{array}$ & \multirow[t]{2}{*}{ Martín, 2008} & \\
\hline & Titulares escuela, profesores asociados y ayudantes & & \\
\hline & $\begin{array}{l}\text { Personal Docente e Investigador (equivalente a tiempo } \\
\text { completo) }\end{array}$ & Vázquez, 2011 & \\
\hline & Número de profesores equivalente a tiempo completo & Larrán y García, 2015 & \\
\hline & Número de profesores equivalente a tiempo completo & García y Larrán, 2008 & \\
\hline & Número de docentes (Tiempo Completo) & Ayaviri y Zamora, 2016 & \\
\hline $\begin{array}{l}\text { NÚMER0 TOTAL DE } \\
\text { TRABAJADORES } \\
\text { ADMINISTRATIVOS (NTA) }\end{array}$ & Número de personal no académico. & $\begin{array}{l}\text { Avkiran, 2001; Abbott y } \\
\text { Doucouliagos, 2003; Parrellada y Duch, } \\
\text { 2006; Duch, 2006. }\end{array}$ & $\begin{array}{l}\text { Varios estudios al respecto, } \\
\text { existe aproximación. }\end{array}$ \\
\hline Outputs & Investigaciones & Autores & $\begin{array}{c}\text { Cuántos estudios al } \\
\text { respecto para la variable } \\
\text { aplicada } \\
\end{array}$ \\
\hline & Número de alumnos matriculados en cada Universidad & Larrán y García, 2015 & \multirow{6}{*}{$\begin{array}{l}\text { Varios estudios al respecto, } \\
\text { existe aproximación. }\end{array}$} \\
\hline \multirow{5}{*}{$\begin{array}{l}\text { NÚMER0 TOTAL DE } \\
\text { MATRICULADOS (NTM) (Hombres, } \\
\text { Mujeres y Total) }\end{array}$} & Cantidad de inscripciones de pregrado & Cáceres, Kristjan y Tabilo, 2013 & \\
\hline & Número de matriculados de $1^{\circ}$ y $2^{\circ}$ ciclo & Martín, 2008 & \\
\hline & $\mathrm{N}^{0}$ de estudiantes matriculados & Ramirez y Alfaro, 2013 & \\
\hline & $\begin{array}{l}\text { Número de alumnos matriculados en centros propios en } \\
1^{\circ} \text { y } 2^{\circ} \text { ciclo }\end{array}$ & $\begin{array}{l}\text { Ahn, Charnes y Cooper, 1988; } \\
\text { Parrellada y Duch, 2006; Duch, } 2006\end{array}$ & \\
\hline & Número de alumnos matriculados en cada Universidad & García y Larrán, 2008 & \\
\hline \multirow{7}{*}{$\begin{array}{l}\text { NÚMER0 TOTAL DETITULADOS } \\
\text { (NTT) (Hombres, Mujeres y Total) }\end{array}$} & $\mathrm{N}^{\circ}$ de alumnos graduados & $\begin{array}{l}\text { Ahn y Seiford, 1993; Coelli, Prasada y } \\
\text { Battesse, 1998; Avkiran, 2001; Abbott } \\
\text { y Douconliagos, 2003; Johnes et al, } \\
\text { 2005; Thanasoullis et al. } 2011\end{array}$ & \multirow{7}{*}{$\begin{array}{l}\text { Varios estudios al respecto, } \\
\text { existe aproximación. }\end{array}$} \\
\hline & Estudiantes de grado matriculados ETC & $\begin{array}{l}\text { Ahn y Seiford, 1993; Coelli, Prasada y } \\
\text { Battesse, 1998; Avkiran, 2001; Abbott } \\
\text { y Douconliagos, 2003; Johnes et al, } \\
\text { 2005; Thanasoullis et al. } 2011 \\
\end{array}$ & \\
\hline & $\begin{array}{l}\text { Número de titulaciones de segundo ciclo/número de } \\
\text { titulaciones }\end{array}$ & Hernangómezet al, 2007 & \\
\hline & $\mathrm{N}^{0}$ de alumnos graduados en cada universidad & Larrán y García, 2015 & \\
\hline & Número de alumnos graduados en $1^{\circ}$ y $2^{\circ}$ ciclo & Vázquez, 2011 & \\
\hline & $N^{0}$ de graduados & Ayaviri y Zamora, 2016 & \\
\hline & Número de alumnos graduados en cada universidad & García y Larrán, 2008 & \\
\hline $\begin{array}{l}\text { NÚMER0 TOTAL DEMATRICULA } \\
\text { NUEVA (NTMN) } \\
\text { Hombres, Mujeres y Total }\end{array}$ & Matrícula de estudiantes nuevos de pregrado & Cáceres, Kristjan y Tabilo, 2013 & $\begin{array}{c}\text { Segundo estudio, considerando } \\
\text { la variables de Matríucula } \\
\text { Nueva }\end{array}$ \\
\hline
\end{tabular}

Fuente: Elaboración propia 
Autores como: Martín (2008); García y Larrán (2008); Vásquez (2011); Amariles y Soto (2015); Larrán y García (2015); Ayaviri y Zamora (2016); consideran como una variable inputs; el "Número Total de Docentes", donde se toma en cuenta solo a docentes Tiempo Completo y no a los Docentes Tiempo Horario; sin embargo, en la investigación se considera importante incluir a los docentes de tiempo horario, por tanto la la variable aplicada en el caso de las universidades del EPB es "Docentes Tiempo Completo y Tiempo Horario", puesto que ambos tipos de docentes por tipo de contrato desarrollan las mismas actividades académicas, por lo que no sería sujeto a discriminación.

Con relación a la variable "Número Total de Trabajadores Administrativos" ésta presenta una aproximación a la aplicación de la variable "Personal no Académico" utilizada en otras investigaciones;como: Avkiran (2001); Abbott y Doucouliagos (2003); Parrellada y Duch (2006); Duch (2006); citado en Vásquez (2011). Sin embargo la variable aplicada en el estudio considera a "Trabajadores Administrativos Permanentes y Eventuales"; ya que ambos desarrollan actividades administrativas.

Con referencia a las variables outputs, como: "Número Total de Matriculados", fueron utilizadas por García y Larrán (2008); Martín (2008); Larrán y García (2015); Cáceres, Kristjan y Tabilo (2013); Ramírez y Alfaro (2013); Ahn, Charnes y Cooper (1988); Parrellada y Duch (2006); Duch (2006); la variable "Número Total de Titulados"; por Ahn y Seiford (1993); Coelli, Prasada y Battesse (1998); Avkiran (2001); Abbott y Douconliagos (2003); Johnes et al, (2005); Thanasoullis et al. (2011); Hernangómez et al, (2007), Larrán y García, (2015); y la variable "Matrícula Nueva Universitaria"; fue aplicada en sólo un estudio de investigación de Cáceres, Kristjan y Tabilo, 2013.

Esto permite concluir que las variables incluidas en el Modelo Envolvente de Datos (DEA) para la medición de la eficiencia universitaria, es un aporte, y permite observar que dichas variables a su vez pueden explicar las eficiencias, ya que los resultados muestran que existen carreras con altos índices de eficiencia técnica; en promedio existe un incremento de la eficiencia técnica entre el año 2014 y 2015 ; y que el número de alumnos, titulados y docentes son los que determinan los índices altos de eficiencia técnica en las carreras.

\section{References:}

1. Aigner D.J., L. C. (1977). Formulation and estimation of stochastic frontier production function models. Journal of Econometrics 6, 2137.

2. Amariles, J. M., \& Soto-Mejía, J. A. (2015). Análisis de sensibilidad de los resultados del modelo de gestión SUE (Sistema Universitario 
Estatal) basado en el análisis envolvente de datos. Ingeniería Investigación de Operaciones, Vol.17, $N^{\circ} 2,53-64$.

3. Asamblea Constituyente. (2007). Constitucion Politica del Estado Plurinacional de Bolivia. La Paz: Ministracion de Presidencia.

4. Autonomías, M. d. (2013). Agenda Patriótica 2025. La Paz.

5. Autonomías, M. d. (2013). Agenda Patriótica 2025. La Paz.

6. Ayaviri, D., \& Zamora, G. (2016). Medición de la eficiencia en las Universidades. Una propuesta metodológica. Perspectivas, año 19, $N^{o} 37,7-22$.

7. Ayaviri-Nina, V. D., \& Zamora Echenique, G. (2016). Medición de la eficiencia en las Universidades. Una propuesta metodológica. Perspectivas, $N^{o}$ 37, 7-22.

8. Banker R.D., C. W. (1984). Some models for estimating technical and scale inefficiencies in data envelopment analysis. Management Science, 1078-1092.

9. Cáceres V., H., Kristjanpoller R., W., \& Tabilo A., J. (2014). Análisis de la eficiencia técnica y su relación con los resultados de la evaluación de desempeño en una Universidad chilena. Innovar, Vol 24, $N^{\circ} 54,199-217$.

10. Caves D.W., C. L. (1981). Productivity growth, scale economies and capacity utilization in U.S.Railroads, 1955-74. American Economic Review 71, 994-1002.

11. CEUB. (2016). Boletín Estadístico $N^{\circ} 3$ del Sistema de la Universidad Boliviana. La Paz: CEUB.

12. CEUB, C. E. (2013). Estatuto Orgánico de la Universidad Boliviana. La Paz.

13. CEUB, C. E. (2014). Plan Nacional de Desarrollo Universitario 2014 - 2018. La Paz: Secretaria Nacional de Desarrollo Institucional SNDI.

14. CEUB, C. E. (2015). Boletín Estadístico No4; Sistema de la Universidad Boliviana en cifras; Año IV. La Paz: CEUB.

15. CEUB, S. N. (2014). Modelo Académico del Sistema de la Universidad Boliviana 2015 - 2019. La Paz - Bolivia: Comité Ejecutivo de la Universidad Boliviana.

16. Charnes, A. C. (1978). Measuring the efficiency of decision making units. European Journal of Operational Research 2, 429-444.

17. Constitución Política del Estado Plurinacional de Bolivia. (2009). El Alto Bolivia.

18. Coria, M. M. (2011). Eficiencia Técnica de las Universidades Argentinas de Gestión Estatal. Ensayos de Política Económica, $N^{o} 5$, 43-63. 
19. Debreu, G. (1951). The coefficient of resource utilization Econometrica.

20. Ecuador, A. L. (2010). Ley Orgánica de Educación Superior $N^{\circ} 298$. Quito.

21. Educación, M. d. (1990). Ley Orgánica Constitucional de Enseñanza (LOCE) $N^{\circ}$ 18962. Santiago.

22. Educación, M. d. (1996). Ley de Directrices y Bases $N^{\circ} 9396$.

23. Educación, M. d. (2006). Estatuto de la Universidad de Chile, Ley $N^{\circ} 3$. Santiago.

24. Eduviges Morales, I. N. (2008). La educación como elemento fundamental del desarrollo endógeno. Frónesis v.15 n.2 , http://www.scielo.org.ve/scielo.php?script=sci_arttext\&pid=S131562682008000200007.

25. Estrada, A. A. (2007). La legislación sobre la Educación Superior en el Perú Antecedentes, Evolución y Tendencias. Lima - Perú.

26. Farrell, M. J. (1957). The measurement of productive efficiency,. Journal of Royal Statistical Society, 253-290.

27. García, L. V., \& Talancón, H. P. (2008). LA EDUCACIÓN COMO FACTOR DEL DESARROLLO INTEGRAL SOCIOECONÓMICO (1). Contribuciones a las Ciencias Sociales, http://www.eumed.net/rev/cccss/02/vgpt.htm.

28. García-Correas, Á., \& Larrán-Jorge, M. (s.f.). Financiación y Eficiencia de las Universidades Públicas Españolas. Un Estudio Empírico. Recuperado el 23 de Enero de 2017, de https://www.researchgate.net/profile/Manuel_Jorge/publication/2426 14274_FINANCIACION_Y_EFICIENCIA_DE_LAS_UNIVERSID ADES_PUBLICAS_ESPANOLAS_UN_ESTUDIO_EMPIRICO/lin ks/00b4953b7b5d7690f3000000.pdf.

29. González Luis Eduardo, E. Ó. (2008). Calidad de la Educación Superior Concepto y Modelos. Calidad de la Educación, 244-276.

30. Hoz, A. d. (1997). Desarrollo y Validación de un Modelo de Calidad Universitaria como Base para su Evaluación. Revista electrónica de investigación y evaluación. Volumen 3, Numero 1.

31. Jimenes, E. (2006). Tendencias probables de la educació en el siglo XXI. Innovaciuones de negocios. 3 (2), 185-195.

32. Koopmans, T. C. (1951). An analysis of production as an efficient combination of activities. New York.

33. Larrán-Jorge, M., \& García-Correas, Á. (2014). ¿Influyen los modelos de financiación autonómicos en la eficiencia de las universidades públicas españolas? Revista de Contablilidad - Spanish Accounting Review 18 (2), 162-173. 
34. Martín, R. (2008). La Medición de la Eficiencia Universitaria: Una Aplicación del Análisis Envolvente de Datos. Formación Universitaria, Vol 1, $N^{\circ} 2,17-26$.

35. Mendoza, L. (27 de agosto de 2014). http://eju.tv/2014/08/gobiernoentrega-reglamento-para-universidades-privadas-de-bolivia/.

Recuperado el 16 de enero de 2017, de ABI.

36. Ministerio, d. E. (2012). Reglamento General de Universidades Privadas. Gaceta Oficial del Estado Plurinacional, 48.

37. Miranda, A. V. (2001). Educación Superior de Calidad para el Siglo XXI. Revista Educación 25(1), 9-17.

38. Moreno-Brid, J. C. (2009). La educación superior y el desarrollo económico en América Latina. CEPAL - Serie Estudios y perspectivas - México $N^{o} 106,1-45$.

39. Moreno-Brid, J. C., \& Ruiz-Nápoles, P. (2010). La educación superior y el desarrollo económico en América Latina. Revista Iberoamericana de Educación Superior, 171-188.

40. Nación, C. d. (2006). Ley Nacional de Educación $N^{\circ}$ 26.206. Buenos Aires.

41. Nacional, A. (2011). Reglamento de la Ley Orgánica de Educación Superior. Quito.

42. Nacional, A. (2011). Reglamento General a la Ley Orgánica de Eduacación Superior. Quito - Ecuador.

43. Nacional, C. (1995). Ley de Educación Superior $N^{\circ} 24521$. Buenos Aires.

44. Nacional, H. C. (1990). Ley $N^{\circ} 1178$ de Administración y Control Gubernamental. La Paz - Bolivia: Gaceta Oficial.

45. Neira, I. (2010). Educación y desarrollo económico: el papel de la cooperación internacional en el desarrollo del tercer mundo. www.usc.es/economet/aeeadepdf/aeeade47.pdf, 13.

46. Orden Hoz, A. d., Asensio Muñoz, I., Biencinto López, C.-M., \& González. (2007). Archivos Analíticos de Políticas Educativas. Analíticos de Políticas Educativas, Volumen 15, Número 12, 1-60.

47. Pinilla Álvarez, A. (2001). La medición de la eficiencia y la productividad. Madrid: Pirámide.

48. Presidencia, M. d. (21 de Octubre de 2008). Constitución Política del Estado Plurinacional de Bolivia. Constitución Política del Estado. La Paz, La Paz, Bolivia: Ministerio de la Presidencia.

49. Ramírez, P. E., \& Alfaro, J. (2013). Evaluación de la Eficiencia de las Universidades pertenecientes al Consejo de Rectores de las Universidades Chilenas: Resultados de un Análisis Envolvente de Datos. Formación Universitaria, Vol.6, $N^{\circ}$ 3, 31-38.

50. República, P. L. (2014). Ley Universitaria $N^{\circ} 30220$. Lima. 
51. Rescala, C., Devincenzi, G., Rohde, G., \& et.al. (2012). Dos Modelos para determinar la eficiencica de una empresa constructora. TRIM, Vol 5, 21-38.

52. Rolando, E. C., Delimiro, V. C., \& José Mario, A. T. (2015). Eficiencia en las instituciones educativas públicas de la ciudad de Santa Marta (Colombia) mediante "Análisis Envolvente de Datos". Ingenieare, Revista Chilena de Ingeniería, Vol. $23 N^{\circ} 4,579-593$.

53. Secretaría Nacional de Desarrollo Institucional, C. (Junio de 2016). Boletín Estadístico No5. Sistema de la Universidad Boliviana en Cifras. La Paz, La Paz, Bolivia: CEUB.

54. Shephard, R. W. (1953). Cost an Production Functions. USA: Princeton University Press.

55. UNESCO. (2007). La educación, elemento esencial del desarrollo sostenible.

56. UTO, U. T. (2016). Plan Estrategico Institucional 2016 - 2020. Oruro: Universitaria.

57. Vásquez-Rojas, A. M. (2011). Eficiencia Técnica y Cambio de Productividad en la Educación Superior Pública: Un Estudio Aplicado al Caso Español (2000-2009). Madrid, Madrid, España.

58. Visbal-Cadavid, D., Mendoza-Mendoza, A., \& Corredor-Carrascal, K. (2015). Evaluación del desempeño docente mediante el análisis envolvente de datos: un estudio de caso. Entramado, Vol. 11, $\mathrm{N}^{\circ}$, 218-225.

59. Weise, C., \& Laguna, J. L. (2008). La Educación superior en la región andina: Bolivia, Perú y Ecuador. Avaliação da Educação Superior (Campinas), http://www.scielo.br/scielo.php?script=sci_arttext\&pid=S141440772008000200009 \#back6. 